\title{
Association between the incidence of varicella and meteorological conditions in Jinan, Eastern China, 2012-2014
}

\author{
Yunqing Yang ${ }^{1 \dagger}$, Xingyi Geng ${ }^{2 \dagger}$, Xiaoxue Liu², Weiru Wang ${ }^{2}$ and Ji Zhang ${ }^{2 *}$
}

\begin{abstract}
Background: Varicella remains an important public health issue in China. In this study we explored the effect of weather conditions on the incidence of varicella in the temperate city of Jinan, Eastern China during 2012-2014 to inform public health prevention and control measures.

Methods: Data on reported cases of varicella were obtained from National Notifiable Disease Report System. Meteorological data for the same time period were obtained from the Jinan Meteorological Bureau. A negative binomial regression model was used to assess the relationships between meteorological variables and the incidence of varicella. Given collinearity between average temperature and atmospheric pressure, separate models were constructed: one including average temperature without atmospheric pressure, the other including atmospheric pressure but without average temperature. Both models included relative humidity, wind velocity, rainfall, sunshine, and year as independent variables.
\end{abstract}

Results: Annual incidence rates of varicella were 44.47, 53.69, and 46.81 per 100,000 for 2012, 2013, and 2014, respectively. Each increase of $100 \mathrm{~Pa}(\mathrm{hPa})$ in atmospheric pressure was estimated to be associated with an increase in weekly incidence of $3.35 \%(95 \% \mathrm{Cl}=2.94-3.67 \%)$, while a $1{ }^{\circ} \mathrm{C}$ rise in temperature was associated with a decrease of $3.44 \%$ (95 \% Cl=-3.73-3.15\%) in the weekly incidence of varicella. Similarly, a $1 \%$ rise in relative humidity corresponded to a decrease of $0.50 \%$ or $1.00 \%$, a $1 \mathrm{~h}$ rise in sunshine corresponded to an increase of $1.10 \%$ or $0.50 \%$, and a $1 \mathrm{~mm}$ rise in rainfall corresponded to an increase of $0.20 \%$ or $0.30 \%$, in the weekly incidence of varicella cases, depending on the variable considered in the model.

Conclusion: Our findings show that weather factors have a significant influence on the incidence of varicella. Meteorological conditions should be considered as important predictors of varicella incidence in Jinan, Eastern China.

Keywords: Varicella, Meteorological variables, Correlation analysis

\section{Background}

Varicella (commonly knows as chickenpox) is an acute infection caused by the Varicella-zoster virus (VZV) [1]. Varicella is highly contagious with an attack rate of $90 \%$ for close contacts of cases [2]. Primary VZV infection is characterized by a generalized pruritic vesicular rash and subsequent latency in dorsal root ganglia. Shingles is a disease caused by reactivation of VZV infection and a common complication of post-herpetic neuralgia.

\footnotetext{
* Correspondence: zhangji1967@163.com

${ }^{\dagger}$ Equal contributors

${ }^{2}$ Jinan Center for Disease Control and Prevention, Shandong Province

250021, P. R. China

Full list of author information is available at the end of the article
}

Although varicella is generally benign and self-limiting within a week, severe complications, including death, can occur. Immunocompromised children and adults are more likely to develop severe complications [3]. Jinan is the major city of Eastern China, with over 6.95 million registered inhabitants. In Jinan, the incidence of varicella is increasing due to airborne and contact transmission: the incidence rate was 2842 per 100,000 in 2006 [4] public health authorities.

To control and prevent varicella, in 2006 the government of Jinan introduced legislation to include varicella into the list of local reportable diseases. Physicians who diagnose suspected or confirmed varicella cases must 
report these cases to Jinan Centers for Disease Control and Prevention (JNCDC) through the National Notifiable Disease Report System (NNDRS). Separate from the immunization strategy for measles and polio, varicella is not incorporated into the Expanded Program on Immunization (EPI) in China. Instead, it is administered as part of a voluntary vaccination program. Although routine childhood varicella vaccination has been strongly recommended by the government, the incidence of varicella in Jinan has reached a high level in recent years. In 2011, the annual incidence of varicella was 55 per 100,000 , a substantially higher incidence rate than that of Japan $(1.3$ per 100,000$)$ [5], Korea (35 per 100,000) [6] and Taiwan $(32$ per 100,000) [7].

The increasing evidence for rapid global climate change has highlighted the need to examine the relationship between weather variability and infectious diseases incidence. However, the impact of weather fluctuations on varicella is still not well understood and published evidence is inconsistent. For example, a study in Hong Kong found no significant association between temperature and varicella incidence [8], contrary to earlier studies in the West Indies [9] that suggested that temperature was inversely correlated with the incidence of varicella. Studies from Mexico [10] and Taiwan [11] have suggested that temperature is positively associated with varicella incidence. These differences are probably due to variation in weather patterns of the study areas and the different variables considered in the models. A recent study showed that atmospheric pressure and rainfall were positively associated with varicella incidence [12], but no other study has supported these findings. Furthermore, the relationship between varicella incidence and other meteorological variables, such as wind velocity and sunshine, has not yet been estimated. Therefore, there remains an urgent need to investigate these relationships to help prediction of future occurrence and to develop early warning systems for varicella.

This study aimed to describe the epidemiology of varicella in the temperate city of Jinan for 2012-2014 and to explore the association between varicella incidence and various meteorological factors.

\section{Methods}

\section{Study area}

Jinan covers an area of $8177 \mathrm{~km}^{2}$, is situated at $36^{\circ} 4^{\prime}$ north and $117^{\circ} 0^{\prime}$ east, and has over 6.95 million registered inhabitants (from 2010 census data). Jinan is warm and semi-humid with a continental monsoon climate, characterized by drought and wind in spring, warm and rainy summers, cool and dry autumns, and dry and sunny winters. The annual mean temperature was $13.8{ }^{\circ} \mathrm{C}$ and the rainfall $685 \mathrm{~mm}$.

\section{Varicella incidence data}

Since May 2006, the Jinan Ministry of Health has categorized varicella as a Class " $\mathrm{C}$ " infectious disease. According to notifiable infectious disease regulation, all varicella cases are reported to JNCDC through NNDRS using a standard pro forma that includes details of name, sex, age, address, and date of symptom onset. The diagnostic criteria for varicella cases are provided in a guidebook published by the Chinese Ministry of Health [13]. The case definition includes (1) fever and characteristic rash, and/or (2) a four-fold rise in antibody titre, or antigen detected in blood, or genetic material detected by polymerase chain reaction. A recent data quality survey has demonstrated that the data are of high quality in China, with reporting completeness of $99.84 \%$ and accuracy of the information reported of $92.76 \%$ [14]. In this study, we used the number of daily reported varicella cases in Jinan from 1 January 2012 to 31 December 2014.

\section{Meteorological data}

Meteorological data, including daily average temperature $\left({ }^{\circ} \mathrm{C}\right)$, relative humidity $(\%)$, atmospheric pressure $(\mathrm{hPa})$, wind velocity $(\mathrm{m} / \mathrm{s})$, rainfall $(\mathrm{mm})$ and sunshine $(\mathrm{h})$ were obtained from the Jinan Meteorological Bureau (http:// data.cma.gov.cn/). Weather data were measured at a fixed-site station located in the central district of Jinan using standard meteorological instruments (barometers, pressure readings, thermometers, anemometers, actinometers, psychrometers, evaporimeters, and weather vanes). Measurements of temperature, relative humidity, atmospheric pressure, and wind velocity were taken every $3 \mathrm{~h}$ before a daily average was calculated. For rainfall and sunshine the daily total only was calculated.

\section{Statistical analysis}

The annual varicella incidence rate was calculated as the total number of new cases reported between 1 January and 31 December of each year, divided by the total population of the same year. A negative binomial multivariable regression model was used to estimate the associations between meteorological variables and varicella incidence. The negative binomial distribution is a Poisson distribution with an over-dispersion term; this term acts as a random effect that allows additional gamma-distributed variance to be added to the Poisson distribution. Given that data were over-dispersed, we selected a negative binomial model rather than a Poisson model. The outcome of models was the total number of varicella cases grouped according to week of onset. Meteorological variables were modeled using a weekly average or by aggregation.

As a preliminary analysis, the Pearson product-moment correlation coefficient $(r)$ was used to examine the 
relationship between meteorological variables. If any two variables (e.g., weather factor $\mathrm{A}$ and weather factor B) showed a strong correlation $(r>0.85)$, two separate negative binomial regression models were constructed: one including weather factor A but not weather factor B, the other including weather factor B but not weather factor A. Both models included all other weather factors as independent variables.

To quantify the effects of meteorological variables, influences $\left(\left[e^{\beta}-1\right]^{*} 100\right)$ were estimated, corresponding to the percentage increase in varicella incidence. The final model included only those variables that reached statistical significance in the model. However, to control for yearly fluctuation, a variable for calendar year was included in the final model irrespective of statistical significance. We used the natural log of predicted rate to examine the linearity between predictor and continuous variables. In addition, residuals were checked using Pearson's goodness of fit. All analyses were carried out using SAS (V.8.01, SAS Institute, Cary, New Jersey, USA). $P$ values $<0.05$ were considered to be statistically significant.

\section{Results}

From 1 January 2012 to 31 December 2014, a total of 10,068 varicella cases were reported in Jinan, China, of which $56.17 \%$ (5655) were male and $43.83 \%$ (4413) female. The highest number of cases was in the age group of $0-5$ years, which accounted for $66.16 \%$ (6661) of the total cases. Annual incidence rates were 44.47, 53.69, and 46.81 per 100,000 , in 2012, 2013, and 2014, respectively. Monthly changes in the number of cases indicated that varicella cases were detected throughout the year. A large peak in the number of cases occurred in November-January where $39.03 \%$ of all cases were reported. A smaller peak occurred in April-May where the number of cases reported accounted for $20.84 \%$ of total cases (Fig. 1).

As shown in Fig. 2, during the study period, the minimum and maximum temperature was -9.40 and $34.60{ }^{\circ} \mathrm{C}$, respectively with an average temperature of $15.46{ }^{\circ} \mathrm{C}$. Relative humidity ranged from 13.00 to $100.00 \%$, with an average of $56.01 \%$. Atmospheric pressure ranged from $857.70 \mathrm{hPa}$ to $1021.80 \mathrm{hPa}$, with average of $995.79 \mathrm{hPa}$. Wind velocity ranged from $0.80 \mathrm{~m} / \mathrm{s}$ to $8.40 \mathrm{~m} / \mathrm{s}$, with an average of $2.63 \mathrm{~m} / \mathrm{s}$. Daily rainfall ranged from $0 \mathrm{~mm}$ to $71.90 \mathrm{~mm}$, with a cumulative total of $1804.25 \mathrm{~mm}$ for the 3 -year time period. Daily sunshine ranged from 0 to $13.5 \mathrm{~h}$, with a cumulative total of $6383.80 \mathrm{~h}$ for the 3 -year time period.

Analysis revealed strong correlations $(r=-0.87, P<0.01)$ between average temperature and atmospheric pressure (Table 1). Therefore, to avoid potential issues with collinearity, two different models including either temperature

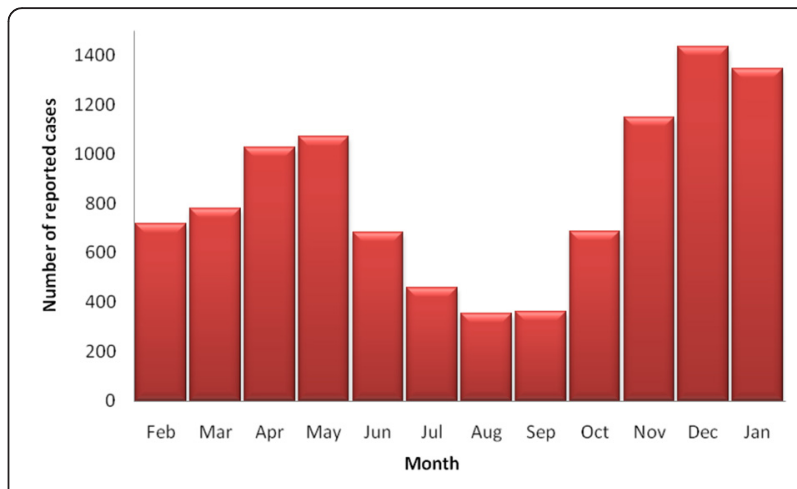

Fig. 1 Monthly distribution of varicella reported cases in Jinan, China, 2012-2014

or atmospheric pressure together with all other predictors were used to explore the relationships between temperature and atmospheric pressure and varicella incidence. In the two models, temperature $(P<0.01)$ and atmospheric pressure $(P<0.01)$ were highly significant; relative humidity, sunshine and rainfall were also statistically significant in both models (all $P<0.05$ ) (Table 2).

After adjusting for calendar year, each $1 \mathrm{hPa}$ rise in atmospheric pressure corresponded to a $3.35 \%$ (95\% $\mathrm{CI}=2.94-3.67 \%)$ rise in weekly varicella incidence, while a $1{ }^{\circ} \mathrm{C}$ rise in temperature corresponded to a decrease of $3.44 \%$ (95\% CI $=-3.73--3.15 \%)$, indicating a reverse effect. Likewise, depending on the variables included in the model, a $1 \%$ rise in relative humidity corresponded to a decrease of $0.50 \%$ or $1.00 \%$ in the weekly incidence of varicella, a $1 \mathrm{~h}$ rise in sunshine corresponded to an increase of $1.10 \%$ or $0.50 \%$, and a $1 \mathrm{~mm}$ rise in rainfall corresponded to an increase of $0.20 \%$ or $0.30 \%$. All models had a good fit (Pearson's chi-square $<0.05$ ).

\section{Discussion}

In recent years, varicella has accounted for significant morbidity and remains a public health issue in China [13]. During our 3-year study period, a total of 10,068 cases were reported, indicating that varicella was highly prevalent in Jinan. Although a highly effective (vaccine effectiveness 80-93\%) varicella vaccine is available and licensed in China, vaccination uptake could be improved if it was part of a universal vaccination program. However, a major obstacle is the high cost of the vaccine, which is 10 times more expensive than the rubella vaccine and 75 times more expensive than the measles vaccine in China [15]. In addition, despite the high incidence, no fatal cases have been reported, which is consistent with other regions of China [16-18], indicating that at present the epidemic status of varicella in Jinan is characterized by high morbidity but low mortality. 


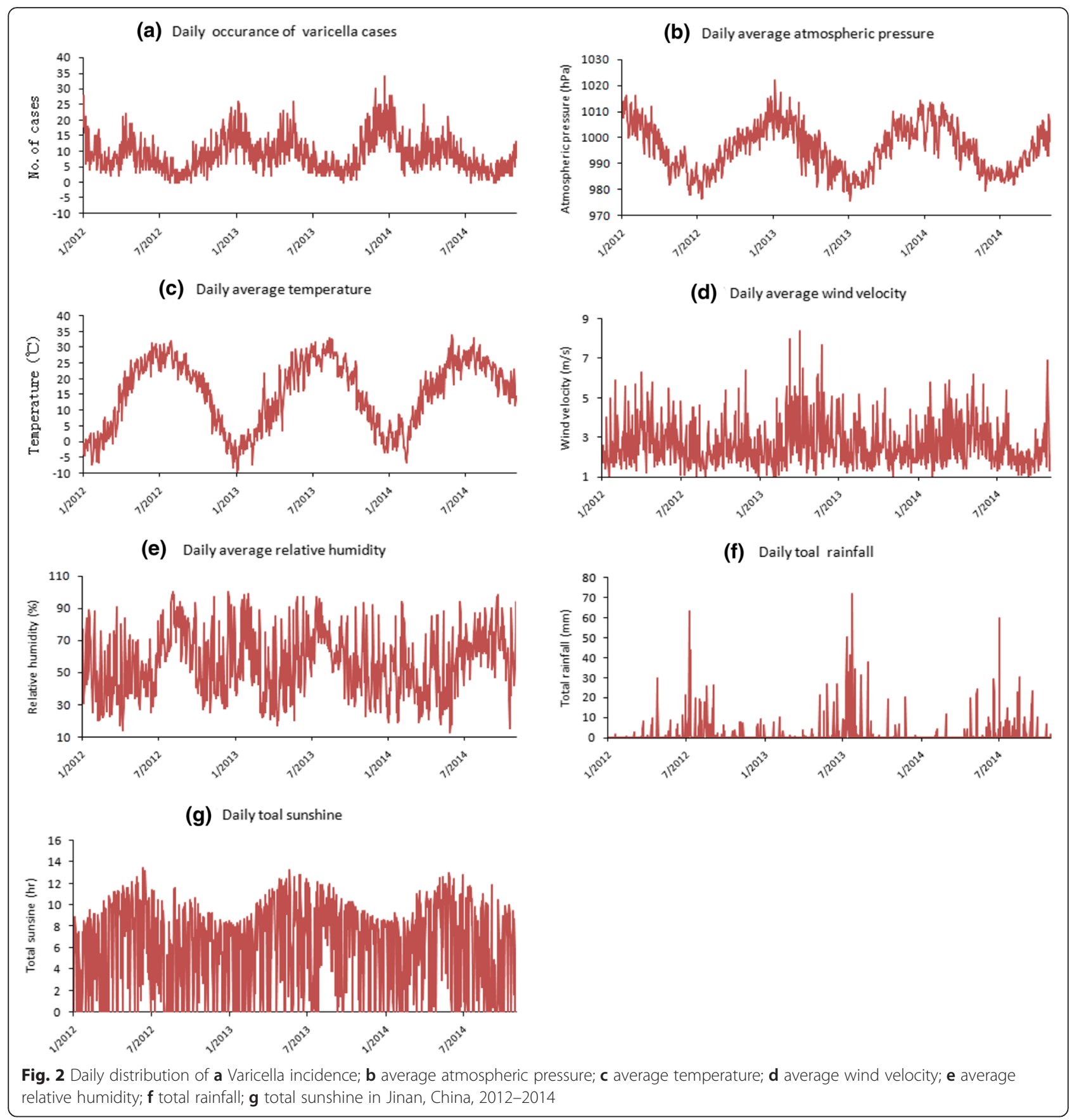

Table 1 Pearson's product-moment correlation coefficient (' $r$ ') matrix for meteorological variables in Jinan, China, 2012-2014

\begin{tabular}{lccccc}
\hline & Relative humidity & Average temperature & Wind velocity & Air pressure & Sunshine \\
\hline Relative humidity & 1.00 & & & & \\
Average temperature & $0.13(P<0.001)$ & 1.00 & & & \\
Wind velocity & $-0.35(P<0.001)$ & $0.08(P=0.01)$ & 1.00 & 1.00 & 1.00 \\
Air pressure & $-0.20(P<0.001)$ & $-0.87(P<0.001)$ & $-0.15(P<0.001)$ & $-0.11(P<0.001)$ & $-0.28(P<0.001)$ \\
Sunshine & $-0.60(P<0.001)$ & $0.21(P<0.001)$ & $0.26(P<0.001)$ & 1.00 \\
Rainfall & $0.37(P<0.001)$ & $0.15(P<0.001)$ & $-0.06(P=0.007)$ & $-0.25(P<0.001)$ & - \\
\hline
\end{tabular}


Table 2 Negative binomial regression model of meteorological factors associated with the incidence of Varicella in Jinan, China, 2012-2014

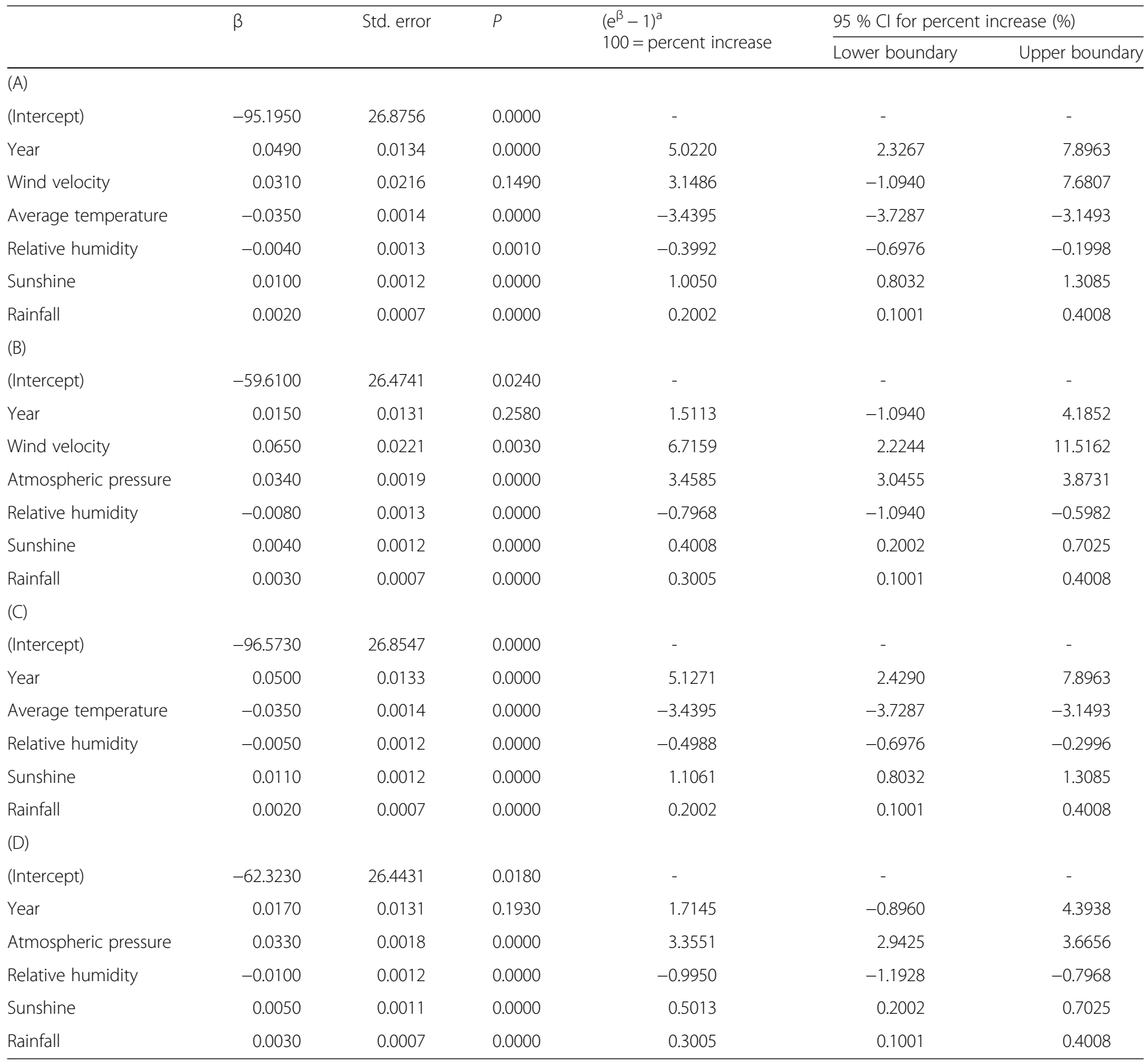

${ }^{a}(A)$ and $(C)$ were respectively preliminary and final models without atmospheric pressure. (B) and (D) were respectively preliminary and final models without average temperature

${ }^{\mathrm{b}} \mathrm{Cl}$, Confidence interval, a type of interval estimate of a population parameter

Similar to findings from Poland [19], Taiwan [7], and other areas of China [20], the majority of varicella cases reported in Jinan were male. This is probably due to more social activities for males whereby they are more likely to be exposed to VZV. Moreover, we found that varicella infection occurred at a very young age; the largest number of reported cases was in the age group of $0-5$ years, which is contrary to the finding in subtropical country of Iraq where most cases were aged 5-14 years (65\%) [21]. Consistent with other areas of China, two peaks of varicella incidence were detected in Jinan: the major peak occurred in November-January, and a minor one in April-May. A similar situation was also observed in Korea [6], located in the same climate zone as Jinan. However, in southern Japan, which has a subtropical monsoon climate, the incidence of varicella was highest in August and lowest in winter [5]. These findings further support the role of climate factors in VZV transmission.

In recent decades, meteorological conditions have been widely studied for their potential as early warning tools to prevent climate-sensitive infectious diseases 
such as fecal-oral infection disease [22], malaria [23], respiratory tract infections [24], and Dengue fever [25]. Our study, demonstrates that weather factors have a significant influence on varicella incidence in Jinan. Particularly, we found that temperature was inversely correlated with varicella incidence. These findings are consistent with those of Garnett et al. who found that in the West Indies fewer varicella infections occur in tropical regions than in temperate regions [9]. In studies from India, Thailand, Sri Lanka, and other countries, varicella incidence has been reported to peak during cooler months [26-28]. A laboratory-based study has suggested that VZV seroprevalence rates in tropical climate are markedly lower in all age groups compared to temperate climates [29]. Furthermore, previous studies have shown that in vitro VZV yield and point of maximum titre were dependent on incubation temperature [30, 31]. Markus et al. demonstrated that VZV replicates more efficiently at lower temperatures [32]. In addition, our study has also indicated that high atmospheric pressure is associated with a higher incidence of varicella. This finding is in general agreement with previous findings from Hong Kong [12] that suggested atmospheric pressure might be considered an important predictor for varicella infection. However, no studies have yet been published revealing the underlying mechanism. A possible explanation might be that high atmospheric pressure is conducive to the spread of virus particles.

We found that relative humidity was inversely correlated with varicella incidence and similar findings have been observed in Hong Kong [8] and the West Indies [9]. The exact mechanism for the potential association between relative humidity and varicella incidence and transmission is unknown. It could hypothesized that in a lower relative humidity, air particles would be smaller and VZV could be suspended for longer in the air and therefore travel over a longer distance, thereby increasing transmission opportunity. Another possible explanation could be that in a dry environment, with more dry skin, patients with varicella suffer from excessive itchiness whereby more skin scratching would facilitate greater viral spread. In addition, we found that a $1 \mathrm{~mm}$ rise in rainfall corresponded to an increase of $0.20 \%$ or $0.30 \%$, in the weekly number of varicella cases in Jinan. This finding is consistent with that of Chan et al. who showed that rainfall at lags of 2-3 days was positively associated with pediatric varicella notification in Hong Kong [12].

To the best of our knowledge, the relationship between sunshine and varicella has not been previously reported. Our study is first to investigate the effect of sunshine on varicella incidence. We found that sunshine was positively associated with varicella incidence. A possible explanation for this might be that longer periods of sunshine correspond to higher doses of solar radiation. Hervás et al. [33] found that the effects of solar radiation, directly on the host immune system and/or indirectly through vitamin D metabolism, may play a role in varicella epidemics [32]. Another possible explanation for this might be that longer periods of sunshine correspond to more time for human activities outdoors where exposure to VZV is more likely.

Some methodological limitations must be acknowledged for this study. First, surveillance data for varicella do not capture all cases in the community. This under-reporting of infections can occur anywhere in the reporting chain, from the initial decision of the patient to seek healthcare, to a failure to record the case in the disease registry due to the mildness or lack of symptoms. Second, although varicella is considered to be an easily recognizable disease $[3,34]$, the notification of varicella cases in our study depended solely on clinical signs, without confirming the diagnosis by microbiological or serological testing, hence resulting in potential misdiagnosis. Third, the incubation period for each case cannot be determined exactly; for this reason we chose to use weekly aggregated data of varicella cases and weekly average/aggregated meteorological data. However, the directions of these approximations are likely to be random, suggesting that our risk estimates are likely reliable. Fourth, weather information obtained from one fixed station might not be representative of the whole city. Finally, owing to this investigation being an ecological study, it is important to note that varicella transmission is multifactorial [35, 36]; besides meteorological factors, other environmental and host factors may also play a role in transmission of VZV. Our current study focused only on the meteorological factors and further studies to incorporate other environmental and host factors, including demographic factors, geographical data, population density, ethnicity, human leukocyte antigen-type predisposition, as well as behavioural data are warranted.

Climatic factors may directly influence the transmission rates of infectious diseased or be associated with changes in host susceptibility. Elucidation of the effects of weather variability on the epidemiology of infectious diseases is becoming increasingly important for disease control by public health officials and practitioners. The results of this study may aid in the prediction of epidemics and in preparation for the effects of climatic change on the epidemiology of varicella through implementation of preventive public health interventions, such as promoting good hygiene practices, temporary closure of educational institutions, active vaccination, and campaigns that include press releases and media events to encourage preventive activities. It is expected that such activities might be practically useful for preventing or limiting the spread of varicella. 


\section{Conclusions}

Varicella remains an important public health concern in Jinan. We have reported the current epidemiological situation for varicella in Jinan, characterized by high morbidity but low mortality. We found that climate parameters should be considered important predictors of varicella activity. A rise in atmospheric pressure, hours of sunshine and rainfall may increase the incidence of varicella, whereas an increase in temperature and relative humidity may reduce the incidence of varicella. Our findings provide preliminary but important information that may be useful for improving understanding of the epidemiology of varicella and help in the development of an early warning system.

\section{Ethics approval and consent to participate}

This study was approved by the ethics committee of Jinan Center for Disease Control and Prevention (JNCDC).

\section{Consent for publication}

Not applicable.

\section{Availability of data and materials}

The raw data will be provided upon request by Dr. Ji Zhang (Correspondence author), Email:zhangji1967@163.com.

\section{Abbreviations}

${ }^{\circ} \mathrm{C}$ : degrees celsius; EPI: expanded program on immunization; JNCDC: Jinan Centre for Disease Control and Prevention; NNDRS: National Notifiable Disease Report System; VZV: varicella zoster virus.

\section{Competing interests}

The authors declare that they have no competing interests.

\section{Authors' contributions}

Conceived and designed the study: JZ. Analyzed the data: XG, YY. Contributed materials/analysis tools: $X G$. Analysis and interpretation of data: $X \mathrm{~L}, \mathrm{WW}$. Wrote the paper: YY. All authors contributed to and approved the final version of the manuscript.

\section{Acknowledgements}

We wish to give special thanks to the public unit coordinators and nurses working in hospitals in Jinan.

\section{Funding}

This study was supported by the project for Medical science and technology of Shandong Province (grant number: 2014WS0009). The funding bodies had no role in the study design, data collection and analysis, preparation of the paper, or the decision to publish.

\section{Author details}

${ }^{1}$ Faculty of Public Health, Shandong University, Shandong Province 250100, P. R. China. ${ }^{2}$ Jinan Center for Disease Control and Prevention, Shandong Province 250021, P. R. China.

Received: 31 March 2015 Accepted: 11 April 2016 Published online: 22 April 2016

\section{References}

1. Breuer J, Whitley R. Varicella zoster virus: natural history and current therapies of varicella and herpes zoster. Herpes. 2007;14 Suppl 2:25-9.

2. Lee BR, Feaver SL, Miller CA, Hedberg CW, Ehresmann KR. An elementary school outbreak of varicella attributed to vaccine failure: policy implications. J Infect Dis. 2004;190(3):477-83.

3. Marchetto S, de Benedictis FM, de Martino M, Versace A, Chiappini E, Bertaine C, Osimani P, Cordiali R, Gabiano C, Galli L. Epidemiology of hospital admissions for chickenpox in children: an Italian multicentre study in the pre-vaccine era. Acta Paediatr. 2007;96(10):1490-3.

4. Liu GF, Song $L Z$, Feng $L$. Investigation on varicella incidence of the children $<$ or $=14$ years old in Shandong province in 2007. Zhongguo Yi Miao He Mian Yi. 2010;16(3):225-8.

5. Toyama N, Shiraki K. Epidemiology of herpes zoster and its relationship to varicella in Japan: a 10-year survey of 48,388 herpes zoster cases in Miyazaki prefecture. J Med Virol. 2009;81(12):2053-8.

6. Cho SB, Oh SH, Ahn BK, Kim HS, Park JM, Lee JH, Lee KH. Incidence of chickenpox in young South Korean soldiers and correlation with atopic dermatitis. Clin Exp Dermatol. 2009:34(6):668-71.

7. Lin YH, Huang LM, Chang IS, Tsai FY, Chang LY. Disease burden and epidemiological characteristics of varicella in Taiwan from 2000 to 2005. J Microbiol Immunol Infect. 2009;42(1):5-12.

8. Chan JY, Tian L, Kwan Y, Chan W, Leung C. Hospitalizations for varicella in children and adolescents in a referral hospital in Hong Kong, 2004 to 2008 : a time series study. BMC Public Health. 2011;11:366.

9. Garnett GP, Cox MJ, Bundy DA, Didier JM, St CJ. The age of infection with varicella-zoster virus in St Lucia, West Indies. Epidemiol Infect. 1993;110(2):361-72.

10. Vergara-Castaneda A, Escobar-Gutierrez A, Ruiz-Tovar K, Sotelo J, Ordonez G, Cruz-Rivera MY, Fonseca-Coronado S, Martinez-Guarneros A, Carpio-Pedroza JC, Vaughan G. Epidemiology of varicella in Mexico. J Clin Virol. 2012;55(1):51-7.

11. Wu PY, Li YC, Wu HD. Risk factors for chickenpox incidence in Taiwan from a large-scale computerized database. Int J Dermatol. 2007:46(4):362-6.

12. Chan JY, Lin HL, Tian LW. Meteorological factors and El Nino Southern Oscillation are associated with paediatric varicella infections in Hong Kong, 2004-2010. Epidemiol Infect. 2014;142(7):1384-92.

13. Li T. Varicella emergency vaccination seemed instrumental in declining chickenpox incident in Guangzhou, Southern China. Rev Inst Med Trop Sao Paulo. 2013:55:3.

14. Li T, Yang Z, Dong Z, Wang M. Meteorological factors and risk of scrub typhus in Guangzhou, southern China, 2006-2012. BMC Infect Dis. 2014;14:139.

15. Ma H, Fontaine R. Varicella outbreak among primary school studentsBeijing, China, 2004. MMWR Morb Mortal Wkly Rep. 2006;55 Suppl 1:39-43.

16. Du Y, Yu F, Zhang L, Wang $X$, Jin B, Wang $Y$, Mei $K$, Lu J, Jiang L. Analysis of protective effect of using chickenpox live attenuated vaccine among 4-17 years old children in Minhang district, Shanghai. Zhonghua Yu Fang Yi Xue Za Zhi. 2014:48(12):1048-52.

17. Zhang X, Yu Y, Zhang J, Huang S, Wang Z, Zhang J, et al. The epidemiology of varicella cases among children in Beijing's Fengtai District from 2008 to 2012. Vaccine. 2014;32(29):3569-72.

18. Lu L, Wang C, Suo L, Li J, Liu W, Pang X, Yan Y, Liu F, Zhao J, He Y. Varicella disease in Beijing in the era of voluntary vaccination, 2007 to 2010. Pediatr Infect Dis J. 2013:32(8):e314-8.

19. Rogalska J, Paradowska-Stankiewicz I. Chickenpox in Poland in 2012. Przegl Epidemiol. 2014:68(2):201-4. 323-324.

20. Hua L, Hongtao $H$, Shunqin W, Jinping G, Jiandong C, Zhaoliang L, Xinwen F. Simultaneous vaccination of Chinese applicants for a United States immigrant visa. Travel Med Infect Dis. 2008;6(3):130-6.

21. Khaleel HA, Abdelhussein HM. Clinical epidemiology of chickenpox in Iraq from 2007-2011. Glob J Health Sci. 2013:5(1):180-6.

22. Li T, Yang Z, Di B, Wang M. Hand-foot-and-mouth disease and weather factors in Guangzhou, southern China. Epidemiol Infect. 2014;142(8):1741-50.

23. Li T, Yang Z, Wang M. Temperature, relative humidity and sunshine may be the effective predictors for occurrence of malaria in Guangzhou, southern China, 2006-2012. Parasit Vectors. 2013;6:155.

24. Li T, Yang $Z$, Wang $M$. Diurnal temperature range may be the risk factor for respiratory tract infections among the elderly in Guangzhou, China. Int Biometeorol. 2014:58(3):309-10.

25. Li TG, Yang ZC, Luo L, Di B, Wang M. Dengue Fever epidemiological status and relationship with meteorological variables in Guangzhou, Southern China, 2007-2012. Biomed Environ Sci. 2013;26(12):994-7. 
26. Lee BW. Review of varicella zoster seroepidemiology in India and Southeast Asia. Trop Med Int Health. 1998;3(11):886-90.

27. Venkitaraman AR, John TJ. The epidemiology of varicella in staff and students of a hospital in the tropics. Int J Epidemiol. 1984;13(4):502-5.

28. Critselis E, Nastos PT, Theodoridou K, Theodoridou M, Tsolia MN, Hadjichristodoulou C, Papaevangelou V. Time trends in pediatric hospitalizations for varicella infection are associated with climatic changes: a 22-year retrospective study in a tertiary Greek referral center. PLoS One. 2012;7(12):e52016

29. Liyanage NP, Fernando S, Malavige GN, Mallikahewa R, Sivayogan S, Jiffry MT, Vitarana T. Seroprevalence of varicella zoster virus infections in Colombo district, Sri Lanka. Indian J Med Sci. 2007;61(3):128-34.

30. Grose C, Perrotta DM, Brunell PA, Smith GC. Cell-free varicella-zoster virus in cultured human melanoma cells. J Gen Virol. 1979;43(1):15-27.

31. Grose C, Brunel PA. Varicella-zoster virus: isolation and propagation in human melanoma cells at 36 and 32 degrees C. Infect Immun. 1978;19(1):199-203.

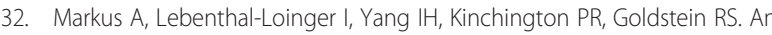
in vitro model of latency and reactivation of varicella zoster virus in human stem cell-derived neurons. PLoS Pathog. 2015;11(6):e1004885.

33. Hervas D, Hervas-Masip J, Nicolau A, Reina J, Hervas JA. Solar radiation and water vapor pressure to forecast chickenpox epidemics. Eur J Clin Microbiol Infect Dis. 2015;34(3):439-46.

34. Wise RP, Salive ME, Braun MM, Mootrey GT, Seward JF, Rider LG, Krause PR. Postlicensure safety surveillance for varicella vaccine. JAMA. 2000;284(10): 1271-9.

35. O'Grady KA, Merianos A, Patel M, Gilbert L. High seroprevalence of antibodies to varicella zoster virus in adult women in a tropical climate. Trop Med Int Health. 2000;5(10):732-6.

36. Brisson M, Edmunds WJ, Law B, Gay NJ, Walld R, Brownell M, Roos L, De Serres G. Epidemiology of varicella zoster virus infection in Canada and the United Kingdom. Epidemiol Infect. 2001;127(2):305-14.

\section{Submit your next manuscript to BioMed Central and we will help you at every step:}

- We accept pre-submission inquiries

- Our selector tool helps you to find the most relevant journal

- We provide round the clock customer support

- Convenient online submission

- Thorough peer review

- Inclusion in PubMed and all major indexing services

- Maximum visibility for your research

Submit your manuscript at www.biomedcentral.com/submit 